\title{
Mitofusin 2 inhibits bladder cancer cell proliferation and invasion via the Wnt/ $\beta$-catenin pathway
}

\author{
GUOFU PANG, QUN XIE and JUANJUAN YAO
}

Department of Urology, Zhuhai People's Hospital, Zhuhai, Guangdong 519000, P.R. China

Received April 15, 2018; Accepted April 12, 2019

DOI: $10.3892 / \mathrm{ol} .2019 .10570$

\begin{abstract}
The present study aimed to investigate the biological role of the mitochondrial GTPase mitofusin-2 (MFN2) in bladder cancer. MFN2 mRNA expression in tumor and paired adjacent non-tumor tissues from 8 patients was investigated using reverse transcription-quantitative polymerase chain reaction analysis. Immunohistochemistry was used to investigate MFN2 expression in 117 bladder cancer specimens. The associations of MFN2 expression with clinicopathological parameters were evaluated statistically. In addition, the biological role of MFN2 in the proliferation, migration and invasion of bladder cancer cells was examined. It was identified that MFN2 expression was significantly downregulated in bladder cancer tissues compared with normal tissues. MFN2 expression was associated with tumor stage, tumor grade and lymph node status. Furthermore, patients with low MFN2 expression demonstrated a shorter overall survival time $(\mathrm{P}=0.025)$. MFN2 knockdown by small interfering RNA promoted cancer cell proliferation, migration and invasion in vitro, and enhanced tumor progression in vivo. Mechanistically, MFN2 was revealed to be involved in $\mathrm{Wnt} / \beta$-catenin signaling. In conclusion, MFN2 may serve as a potential therapeutic target in the treatment of bladder cancer, and the progress of bladder cancer may be delayed by regulating MFN2 expression.
\end{abstract}

\section{Introduction}

Bladder cancer is the most common malignancy in the human urinary system (1). Histologically, bladder cancer can be divided into the following types: Non-muscle invasive bladder cancer (NMIBC) and muscle invasive bladder cancer (MIBC) (2). A total of 70-80\% of all cases are diagnosed as NMIBC and $20 \%$ of patients have locally advanced or metastatic disease at the time of diagnosis (3). The long-term

Correspondence to: Dr Qun Xie, Department of Urology, Zhuhai People's Hospital, 79 Kanging Road, Zhuhai, Guangdong 519000, P.R. China

E-mail:drqunxie@sina.cn

Key words: mitofusin 2, bladder cancer, proliferation, migration, invasion disease-free survival rate of patients with advanced stage or metastatic disease is only $15 \%$ (4). Therefore, it is critical to identify novel predictive markers for tumor metastasis to inform the clinical prognosis of patients with bladder cancer.

The mitochondrial GTPase mitofusin-2 (MFN2) is a GTPase embedded in the outer membrane of mitochondria. The MFN2 gene is located at chromosome 1p36.22 and is composed of 757 amino acids (5). MFN2 participates in mitochondrial fusion and contributes to the maintenance and operation of the mitochondrial network (6). Additionally, MFN2 has been demonstrated to participate in numerous physiological processes, including mitochondrial fusion, cardiac metabolism and the mitochondria-mediated apoptotic pathway (7). Further studies have indicated that MFN2 is involved in the regulation of vascular smooth muscle cell proliferation, and it may serve a role in the pathophysiology of obesity and Charcot-Marie-Tooth disease $(8,9)$. MFN2 has been implicated in a number of human cancer types to serve a role in driving proliferation and metastasis. For example, $\mathrm{Xu}$ et al (10) demonstrated that MFN2 suppresses breast cancer cell progression by inhibiting mTOR complex 2 (mTORC2)/Akt signaling. Zhang et al (11) revealed that MFN2 overexpression can suppress gastric cancer cell proliferation and colony formation, weaken the invasion and migratory ability of cancer cells by downregulating matrix metalloproteinase (MMP)-2 and MMP-9, inhibit the cell cycle and induce apoptosis. However, the biological roles of MFN2 in bladder cancer remain unknown.

The current study evaluated the expression of MFN2 in bladder cancer tissues and cells. Subsequently, the associations between MFN2 expression, clinical pathological factors and prognosis of patients with bladder cancer were evaluated. Furthermore, the role of MFN2 in bladder cancer cell proliferation, migration and invasion was investigated. In summary, the results of the present study suggested that MFN2 serves a crucial role in bladder cancer development and progression.

\section{Materials and methods}

Patients. For the present retrospective study, 117 patients (55 male and 62 female patients aged from 42-72 years) with MIBC were recruited at Zhuhai People's Hospital (Zhuhai, China) between January 2007 and June 2012. Patients who had received chemotherapy prior to surgery were excluded from the present study. Tumor stages were determined according to 
the criteria of the World Health Organization (12). In addition, eight pairs of snap-frozen bladder cancer and normal adjacent-noncancerous tissues ( 5 male and 3 female patients aged from 45-67 years) for reverse transcription-quantitative polymerase chain reaction (RT-qPCR) analysis were obtained from Zhuhai People's Hospital between January 2012 and June 2012. The present study was approved by the Institutional Review Board of Zhuhai People's Hospital. Written informed consent was obtained from all participants in the study.

Gene set enrichment analysis (GSEA). Data were obtained from the cancer genome atlas hub (https://tcga.xenahubs.net, data set ID: TGGA, BLCA.sampleMap/ HiSeqV2_PANCAN). GSEA was performed using the GSEA platform (version 2.0.9; http://www. broadinstitute.org/gsea) to assess the association between MFN2 expression and Wnt signaling pathway signatures.

Cell lines and transfection. The bladder cancer cell lines, T24 and J82, were obtained from The Type Culture Collection of the Chinese Academy of Sciences (Shanghai, China). The bladder cancer cell lines T24 and J82 were grown in RPMI-1640 medium (Invitrogen; Thermo Fisher Scientific, Inc., Waltham, MA, USA) supplemented with $10 \%$ fetal bovine serum (FBS; HyClone; GE Healthcare Life Sciences, Logan, UT, USA) at $37^{\circ} \mathrm{C}$ in $5 \% \mathrm{CO}_{2}$. The control scramble small interfering (si)RNA and the si-MFN2 were synthesized by Shanghai GenePharma Co., Ltd. (Shanghai, China). Cells were transfected with control scramble siRNA or the si-MFN2 isoforms at a final concentration of $1 \mathrm{mg} / \mathrm{ml}$ and $100 \mathrm{nM}$, respectively, using Lipofectamine ${ }^{\circledR}$ (Invitrogen; Thermo Fisher Scientific, Inc.), according to the manufacturer's protocol. Subsequent experiments were performed $48 \mathrm{~h}$ after transfection.

$R T$ - $q P C R$. Total RNA from cells and tissue samples were extracted using TRIzol reagent (Invitrogen; Thermo Fisher Scientific, Inc.), followed by the reverse transcription of RNA into cDNA using PrimeScript ${ }^{\mathrm{TM}}$ RT Master Mix (Takara Biotechnology Co., Ltd., Dalian, China) according to the manufacturer's protocol. PCR amplifications were performed using a SYBR PCR kit (Applied Biosystem; Thermo Fisher Scientific, Inc.) RT-qPCR was performed using the StepOnePlus Real-Time PCR system (Applied Biosystems; Thermo Fisher Scientific, Inc.). The sequences of primers are presented in Table I The transcripts were amplified with an initial denaturation at $95^{\circ} \mathrm{C}$ for $30 \mathrm{sec}$, followed by 40 cycles at $95^{\circ} \mathrm{C}$ for $5 \mathrm{sec}, 55^{\circ} \mathrm{C}$ for $30 \mathrm{sec}$ and $72^{\circ} \mathrm{C}$ for $34 \mathrm{sec}$. MFN2 expression data were normalized to GAPDH expression. Relative mRNA expression levels were calculated using the $2^{-\Delta \Delta \mathrm{Cq}}$ method (13). The map for the Wnt/ $\beta$-catenin genes was generated using $\mathrm{MeV}$ 4.9.0 software (14).

Western blot analysis. Both cell lines were lysed using radioimmunoprecipitation assay lysis buffer (Shanghai Biyunyian Bio-Tech Co., Ltd., Shanghai, China) at $4^{\circ} \mathrm{C}$ for $1 \mathrm{~h}$. The protein concentration of each lysate was determined using a bicinchoninic acid protein assay kit (Thermo Fisher Scientific, Inc.) Protein samples (30 $\mu \mathrm{g} /$ lane) were electrophoresed using $8-15 \%$ SDS-PAGE, followed by transfer to
Table I. Reverse transcription-quantitative polymerase chain reaction primer sequences.

\begin{tabular}{|c|c|}
\hline Gene & Primers $\left(5^{\prime} \rightarrow 3^{\prime}\right)$ \\
\hline \multirow[t]{2}{*}{ MFN2 } & F: CCCCCTTGTCTTTATGCTGATGTT \\
\hline & R: TTTTGGGAGAGGTGTTGCTTATTTC \\
\hline \multirow[t]{2}{*}{ CCND1 } & F: CAATGACCCCGCACGATTTC \\
\hline & R: CATGGAGGGCGGATTGGAA \\
\hline \multirow[t]{2}{*}{ CTLA4 } & F: CATGATGGGGAATGAGTTGACC \\
\hline & R:TCAGTCCTTGGATAGTGAGGTTC \\
\hline \multirow[t]{2}{*}{ c-myc } & F: GGCTCCTGGCAAAAGGTCA \\
\hline & R: CTGCGTAGTTGTGCTGATGT \\
\hline \multirow[t]{2}{*}{ c-Jun } & F: TCCAAGTGCCGAAAAAGGAAG \\
\hline & R: CGAGTTCTGAGCTTTCAAGGT \\
\hline \multirow[t]{2}{*}{ LEF1 } & F: ATGTCAACTCCAAACAAGGCA \\
\hline & R: CCCGGAGACAAGGGATAAAAAGT \\
\hline \multirow[t]{2}{*}{ TCF1 } & F: TTGATGCTAGGTTCTGGTGTACC \\
\hline & R: CCTTGGACTCTGCTTGTGTC \\
\hline \multirow[t]{2}{*}{ AXIN2 } & F: ACTGCCCACACGATAAGGAG \\
\hline & R: CTGGCTATGTCTTTGGACCA \\
\hline \multirow[t]{2}{*}{ GAPDH } & F: TCAAGAAGGTGGTGAAGCAG \\
\hline & R: CGTCAAAGGTGGAGGAGTG \\
\hline
\end{tabular}

MFN2, mitofusin 2; CCND1, cyclin D1; CTLA4, cytotoxic T-lymphocyte associated protein 4; lymphoid enhancer binding factor 1; TCF1, T-cell factor 1 .

polyvinylidene difluoride membranes. Following blocking of non-specific binding for $1 \mathrm{~h}$ using 5\% non-fat milk at room temperature., the membranes were incubated overnight on ice with primary antibodies against MFN2 (1:2,000; cat. no. ab124773; Abcam, Cambridge, UK), $\beta$-catenin (1:4,000; cat. no. ab32572; Abcam), GAPDH (1:2,500; cat. no. ab70699; Abcam) and p84 (1:2,500; cat. no. ab125019; Abcam). Subsequently, the membranes were incubated with horseradish peroxidase-conjugated anti-rabbit secondary antibody (1:4,000; cat. no. ab6721; Abcam) for $2 \mathrm{~h}$ at room temperature. A membrane-enhanced chemiluminescence reagent ECL kit (GE Healthcare Life Sciences) was used to visualize the immunoreactive bands.

Immunohistochemical (IHC) analysis. Tissues were fixed in $4 \%$ paraformaldehyde solution at room temperature for $24 \mathrm{~h}$ and embedded in paraffin and serial sections ( $4 \mu \mathrm{M}$ thick) were prepared. Sections were de-paraffinized in dimethylbenzene I, II and IIII solutions, each for $5 \mathrm{~min}$, rehydrated with a gradient of alcohol solutions $(100,95,75$ and $50 \%$, each for $5 \mathrm{~min}$ ) and the endogenous peroxidase activity was blocked using $3 \%$ hydrogen peroxide for $15 \mathrm{~min}$. The section was pre-treated using pressure cooker $\left(100^{\circ} \mathrm{C}\right)$ heat-mediated antigen retrieval with sodium citrate buffer $(\mathrm{pH}$ 6.0) for $30 \mathrm{~min}$. Non-specific immunoglobulin binding was blocked by incubation with $10 \%$ normal goat serum (cat. no. ab7481, Abcam) for $15 \mathrm{~min}$. Following washing with phosphate-buffered saline (PBS), the slides were incubated with a primary 
A

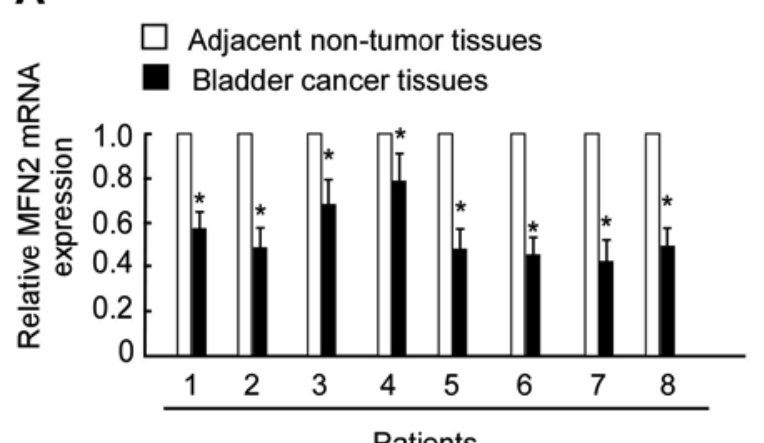

C

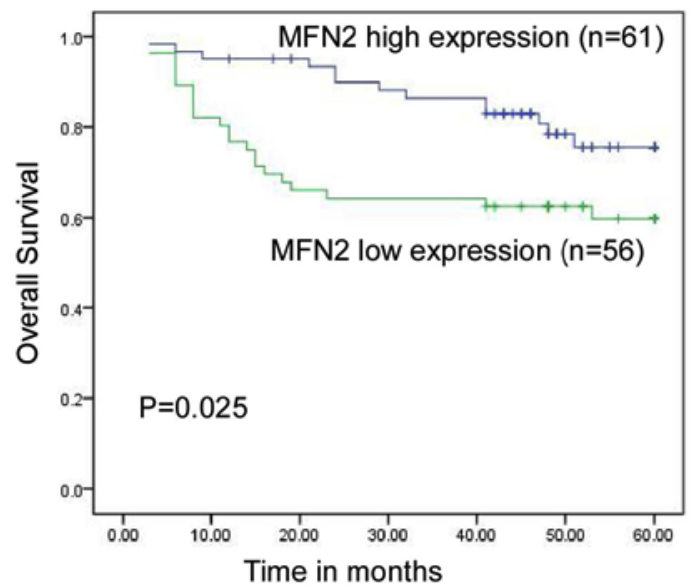

Patients
B
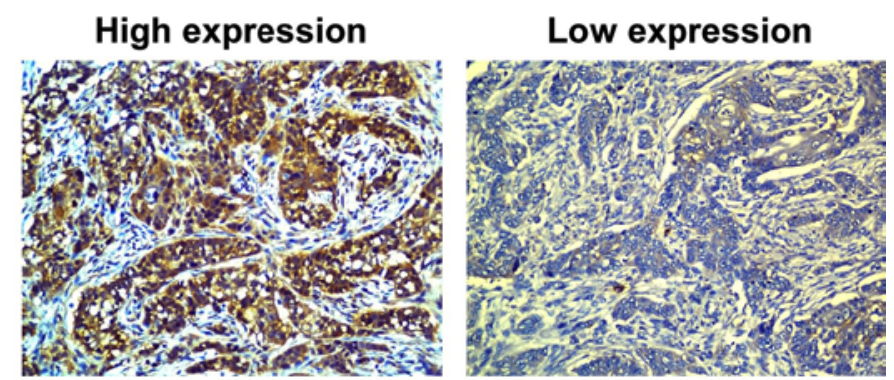

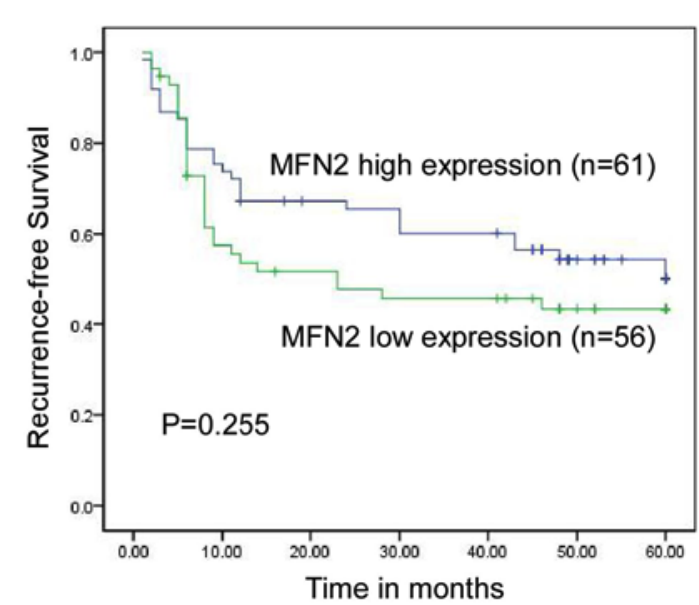

Figure 1. MFN2 expression level in bladder cancer. (A) The expression level of MFN2 mRNA is significantly lower in bladder cancer tissues compared with normal bladder tissues, as determined by reverse transcription-quantitative polymerase chain reaction. (B) Representative immunohistochemical staining of MFN2 in bladder cancer tissues. Magnification, x400. (C) Kaplan-Meier survival curves of overall survival (left) and recurrence-free survival (right) for patients with bladder cancer according to MFN2 expression level. Both overall survival and recurrence-free survival were significantly higher in patients with high levels of MFN2 expression compared with patients with low levels of MFN2 expression. Data are presented as the mean \pm standard deviation "P $<0.05$ vs. respective adjacent non-tumor tissue group. MFN2, mitofusin 2.

antibody against MFN2 (1:200; cat. no. ab124773; Abcam) or Ki67 (1:200; cat. no. ab15580; Abcam) for $60 \mathrm{~min}$ at room temperature, followed by a biotin-conjugated secondary antibody (1:1,000; cat. no. ab6720, Abcam) for $30 \mathrm{~min}$ at room temperature. Slides were observed under a light microscope (magnification, x40). The intensity of MFN2 staining was scored as follows: 0 , negative staining; 1, weakly positive; 2 , moderately positive; and 3 , strongly positive. The percentage of staining was scored as follows: $0,0 \%$ immunoreactive cells; $1, \leq 5 \%$ immunoreactive cells; $2,5-50 \%$ immunoreactive cells; $3,51-80 \%$ immunoreactive cells; and $4,>80 \%$ immunoreactive cells. The IHC score was multiplied by the intensity percentage of staining. Scores of $0-4$ were considered to indicate 'low' expression and those of 6-12 were considered to indicate 'high' expression.

Bromodeoxyuridine labeling. A total of $5 \times 10^{4}$ cells (T24 or J82) were grown on coverslips at $70 \%$ confluence were incubated with 5-bromodeoxyuridine (BrdU) for $1 \mathrm{~h}$ and stained with anti-BrdU antibody (1:250; cat. no. BRDUAPCMOBU-1; Upstate Biotechnology, Inc., Lake Placid, NY, USA) at room temperature, according to the manufacturer's protocol. Cells were stained with DAPI ( $1 \mu \mathrm{g} / \mathrm{ml}$; Sigma-Aldrich; Merck $\mathrm{KGaA}$ ) for $20 \mathrm{~min}$ at room temperature. All images were captured via fluorescence microscopy (magnification, x20).
ImageJ 1.47 (National Institutes of Health, Bethesda, MD, USA) was used to analyze the resulting images.

Wound healing assay. Both cell lines were plated in 24-well plates and serum-starved for $24 \mathrm{~h}$ prior to scratching with a pipette tip. Following scratching, the cells were washed with PBS, images were obtained using a light microscope (magnification, x20) and the cells were placed into medium with $1 \% \mathrm{FBS}$ to prevent proliferation. After incubating the cells at $37^{\circ} \mathrm{C}$ in a $5 \% \mathrm{CO}_{2}$ atmosphere. Wound closure was calculated by examining the distance between the opposite edges of the wound and normalizing the width to the starting width using a light microscope (magnification, x20). Each sample was assayed in triplicate and Image J 1.47 was used to analyze the resulting images.

Cell invasion and migration assay. Cell invasion and migration ability was detected using a Transwell system (EMD Millipore, Billerica, MA, USA). Following transfection with MFN2-specific siRNA, $1 \times 10^{4}$ of both cell lines in $200 \mu 1$ serum-free Dulbecco's modified Eagle's medium (DMEM; HyClone; GE Healthcare, Chicago, IL, USA) were seeded in the upper chamber of a Transwell and the bottom of the chamber was filled with $600 \mu \mathrm{l}$ DMEM containing $10 \%$ FBS. For the invasion assay, Matrigel (1:5; Becton, Dickinson and 
Company, Franklin Lakes, NJ, USA) was added to pre-coat the Transwell chambers and incubated at $37^{\circ} \mathrm{C}$ for $3 \mathrm{~h}$. Cells on the upper side of the filter were removed after incubating the cells at room temperature for $24 \mathrm{~h}$. The filter membrane was stained with crystal violet for $15 \mathrm{~min}$ at $25^{\circ} \mathrm{C}$ and the number of the cells that remained adherent to the underside of the membrane were counted using an inverted light microscope (magnification, $\mathrm{x} 20$ ).

Dual-luciferase reporter assay. Both cell lines were plated in triplicate wells of 24-well plates and allowed to settle for $12 \mathrm{~h}$. Subsequently, $100 \mathrm{ng}$ of reporter plasmids (100 ng; EMD Millipore, Billerica, MA, USA) containing TOP-Flash or FOP-Flash luciferase reporter plus $1 \mathrm{ng}$ pRL-TK Renilla plasmid were co-transfected using Lipofectamine ${ }^{\circledR} 2000$ (Invitrogen; Thermo Fisher Scientific, Inc.). Firefly and Renilla luciferase activity were measured $48 \mathrm{~h}$ following transfection using a Dual Luciferase Reporter assay kit (Promega Cooperation, Madison, WI, USA), according to manufacturer's protocol.

Tumor models. All experimental procedures were approved by the Institutional Animal Care and Use Committee of Zhuhai People's Hospital. Male five-week-old BALB/c nude mice (14-16 g) were purchased from Shanghai SLAC Laboratory Animal Co.,Ltd. (Shanghai, China). The mice were maintained in a pathogen-free environment with free access to food/water, on a 10/14 h light/dark cycle, with an ambient temperature between 18 and $29^{\circ} \mathrm{C}$ and a daily temperature difference $\leq 3^{\circ} \mathrm{C}$, relative humidity $40-70 \%$ and the air ventilated 10 times $/ \mathrm{h}$. For the tumor formation assay, $5 \times 10^{6}$ cells of either cell line in suspension were subcutaneously injected into the flank of each mouse. Tumor volume was measured every 3 days and calculated with the following formula: Length $\mathrm{x}$ width ${ }^{2} / 2$. After 30 days, the mice were deeply anesthetized by an intraperitoneal injection with $1 \%$ sodium pentobarbital $(50 \mathrm{mg} / \mathrm{kg}$; cat. no. 57-33-0; Sigma-Aldrich; Merck KGaA) and sacrificed by cervical dislocation. Death was confirmed by heart beat assessment through direct cardiac palpation. The tumors were detected using an in vivo imaging system (Lumina III, PerkinElmer Co., Ltd., USA).

Statistical analysis. SPSS 16.0 (SPSS, Inc., Chicago, IL, USA) was used for statistical analysis. All data were derived from a minimum of three independent experiments and are presented as the mean \pm standard deviation. Survival curves were generated using the Kaplan-Meier method and the differences were compared using a log-rank test. Multivariate analysis was performed using the Cox proportional hazard regression model. Differences between groups were assessed using one-way analysis of variance followed by Tukey's post hoc analysis. $\mathrm{P}<0.05$ was considered to indicate a statistically significant difference.

\section{Results}

Expression of MFN2 in bladder cancer tissues. MFN2 mRNA expression levels were determined in eight pairs of bladder cancer and corresponding adjacent non-tumor tissues by RT-qPCR. The results demonstrated that MFN2 mRNA
Table II. Associations between MFN2 expression and clinicopathological parameters of patients with bladder cancer.

\begin{tabular}{|c|c|c|c|}
\hline \multirow[b]{2}{*}{ Variable } & \multicolumn{2}{|c|}{ MFN2 expression } & \multirow[b]{2}{*}{ P-value } \\
\hline & Low $(n=56)$ & High $(n=61)$ & \\
\hline Age, years & & & 0.357 \\
\hline$<50$ & 22 & 19 & \\
\hline$\geq 50$ & 34 & 42 & \\
\hline Sex & & & 0.173 \\
\hline Male & 30 & 25 & \\
\hline Female & 26 & 36 & \\
\hline Tumor stage & & & 0.006 \\
\hline I-II & 18 & 35 & \\
\hline III-IV & 38 & 26 & \\
\hline Tumor size, $\mathrm{cm}$ & & & 0.072 \\
\hline$\leq 3$ & 21 & 33 & \\
\hline$>3$ & 35 & 28 & \\
\hline Tumor grade & & & 0.037 \\
\hline Low & 27 & 41 & \\
\hline High & 29 & 20 & \\
\hline Histopathological type & & & 0.814 \\
\hline TCC & 44 & 49 & \\
\hline Other & 12 & 12 & \\
\hline Lymph node status & & & 0.002 \\
\hline Negative & 39 & 56 & \\
\hline Positive & 17 & 5 & \\
\hline
\end{tabular}

MFN2, mitofusin 2; TCC, transitional cell carcinoma.

expression was significantly lower in bladder cancer compared with non-tumor hepatic tissues (Fig. 1A). Representative immunostaining of MFN2 in bladder cancer tissues is presented in Fig. 1B.

Associations between MFN2 expression and clinicopathological factors. The clinical significance of the MFN2 expression level in patients with bladder cancer is summarized in Table II. Tumor stage $(\mathrm{P}=0.006)$, tumor grade $(\mathrm{P}=0.037)$ and lymph node status $(\mathrm{P}=0.002)$ were identified to be significantly associated with MFN2 expression, however, no associations were revealed for age, sex, tumor size or histological type. Furthermore, Kaplan-Meier survival curves demonstrated that patients with a low MFN2 expression level exhibited a markedly worse overall survival time compared with patients with high MFN2 expression $(\mathrm{P}=0.025)$. However, MFN2 expression did not affect the recurrence-free survival time $(\mathrm{P}=0.255$; Fig. 1C). Multivariate analysis using the Cox proportional hazards model also indicated that MFN2 expression was an independent predictor for patients with bladder cancer $(\mathrm{P}=0.017$; Table III $)$.

MFN2 knockdown promotes bladder cancer cell proliferation and migration. To assess the biological roles of MFN2 in 
A
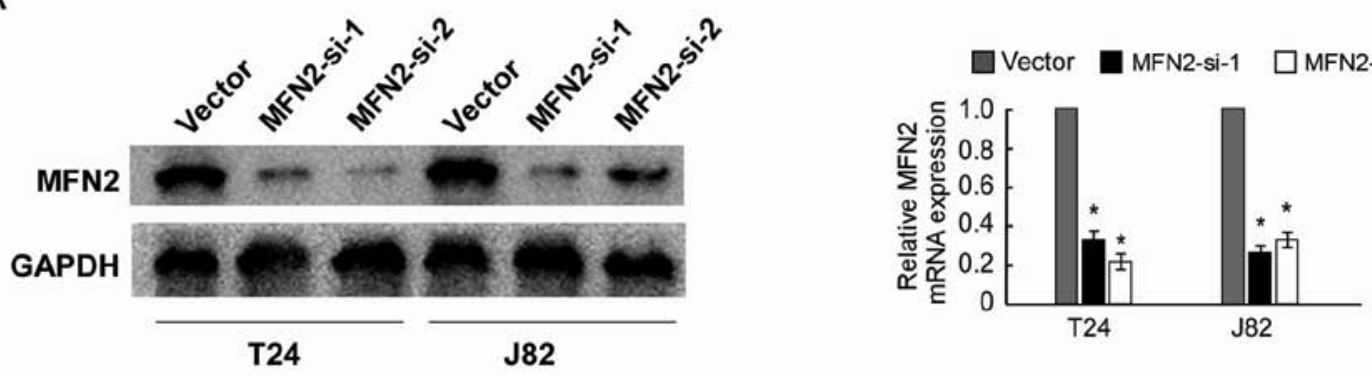

B
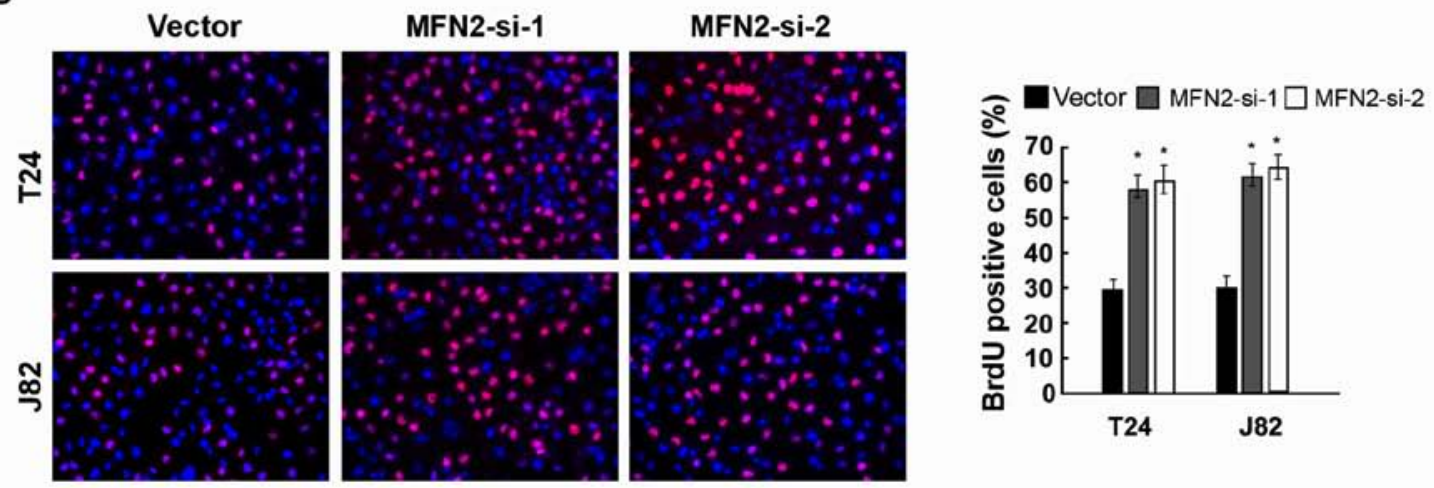

C
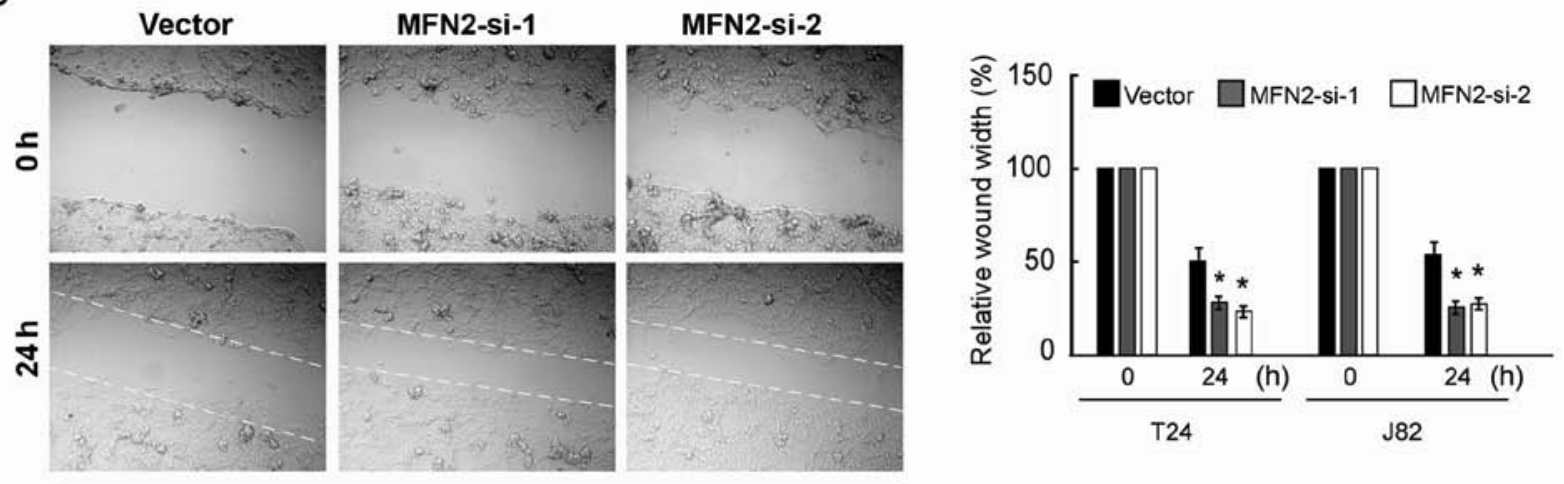

T24

D
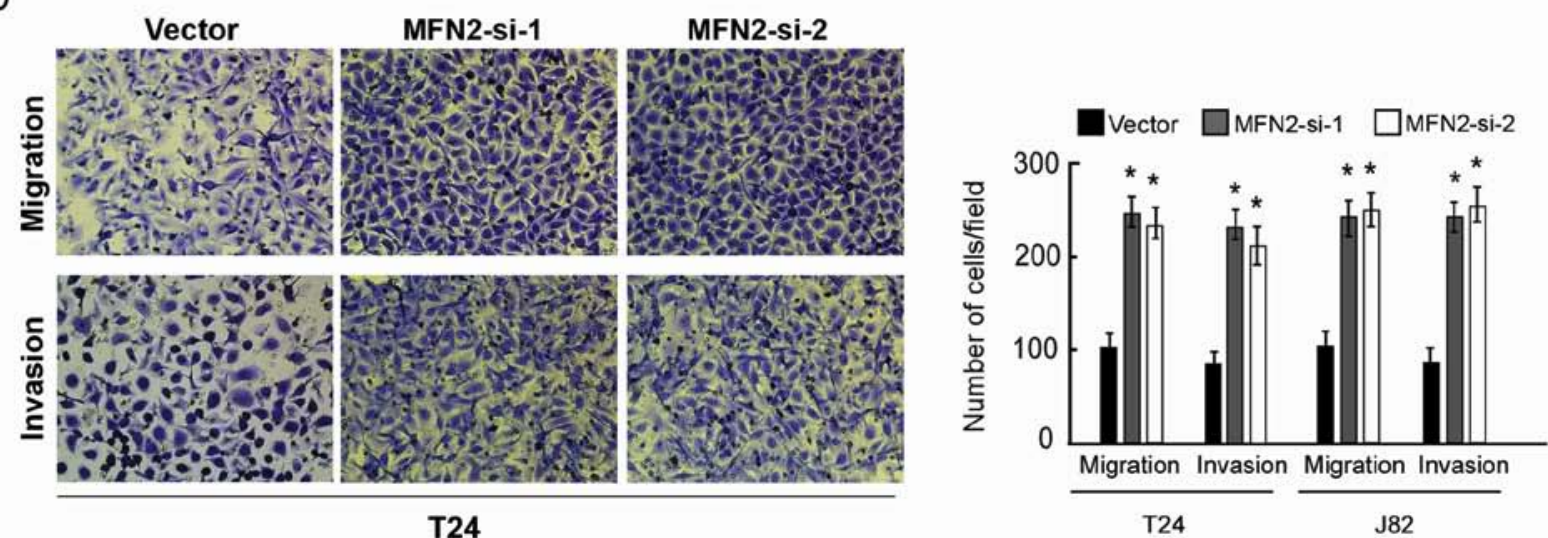

Figure 2. Knockdown of MFN2 promotes the proliferation, migration and invasion of cancer cells. (A) The transfection efficiency of si-MFN2 was analyzed by measuring transcript levels in T24 and J82 cells using reverse transcription-polymerase chain reaction and western blotting. (B) Representative micrographs and quantification (right panel) of BrdU incorporation in the indicated bladder cancer cells. Magnification, x200. (C) The effect of MFN2 knockdown in cancer cell migration was determined by wound healing assay and the uncovered areas in the wound healing assays were quantified as a percentage of the original wound area (right panel). (D) Transwell cell migration assays and Matrigel invasion assays of cancer cells revealed an increased migration and invasive ability of T24 and J82 cells following transfection with si-MFN2. Magnification, x200. Quantification of the migratory and invasive cells of each cell line are presented as proportions of their vector controls (right panels). Data are presented as the mean \pm standard deviation of three independent experiments ${ }^{*} \mathrm{P}<0.05$ vs. respective vector group. MFN2, mitofusin 2; si, small interfering; BrdU, 5-bromodeoxyuridine. 
Table III. Multivariate Cox regression analysis of clinicopathological variables.

\begin{tabular}{lccrc}
\hline & \multicolumn{2}{c}{ OS } & \multicolumn{2}{c}{ RFS } \\
\cline { 2 - 3 } Variable & HR $(95 \%$ CI $)$ & P-value & HR (95\% CI) & P-value \\
\hline Age, years (<50 vs. $\geq 50)$ & - & - & - & - \\
Sex (male vs. female) & - & - & - & - \\
Tumor stage (III-IV vs. I-II) & $2.656(0.892-8.125)$ & 0.022 & - & - \\
Tumor grade, (high vs. low) & - & - & - & - \\
Tumor size, cm (>3 vs. $\leq 3)$ & - & - & - & 0.040 \\
Histological type (TCC vs. other) & - & - & - \\
Lymph nodes status (+ vs. - $)$ & $2.467(0.401-7.377)$ & 0.042 & $2.562(1.154-8.529)$ & - \\
MFN2 expression (low vs. high) & $2.156(0.684-6.452)$ & 0.017 & - & - \\
\hline
\end{tabular}

OS, overall survival; RFS, recurrence-free survival; HR, hazard ratio; CI confidence interval; TCC, transitional cell carcinoma; MFN2, mitofusin 2 .

A

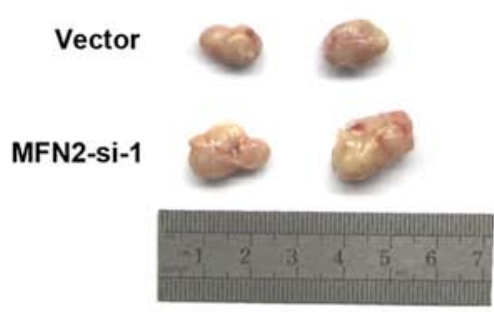

T24
B

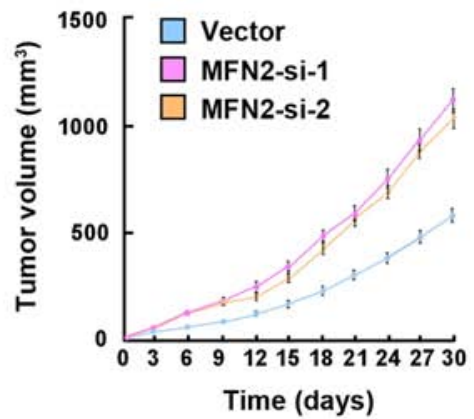

C

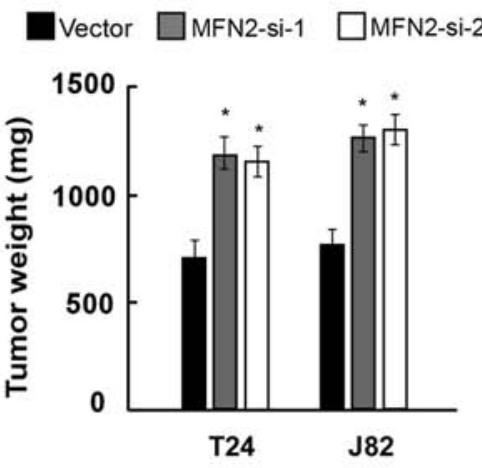

D

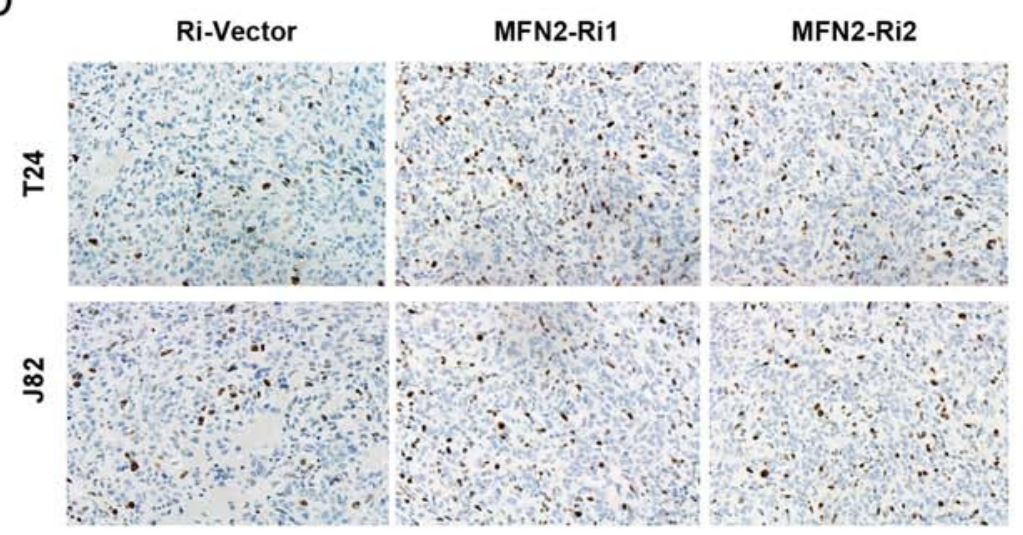

E

Vector $\square$ MFN2-si-1 $\square$ MFN2-si-2

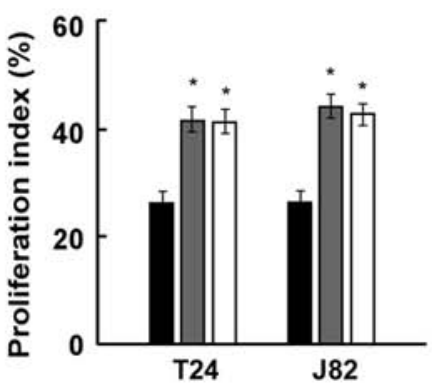

Figure 3. MFN2 knockdown enhances the tumorigenicity of bladder cancer cells. (A) Representative images of the tumors from the xenograft model in nude mice. (B) Tumor volumes were measured on the indicated days. (C) Tumor weights of all mice in each group. (D) Immunohistochemical staining of Ki67 revealed that suppression of MFN2 induced tumorigenicity. Magnification, x200. (E) Quantification of the proliferation index of the indicated cells. Data are presented as the mean \pm standard deviation of three independent experiments. ${ }^{*} \mathrm{P}<0.05$ vs. respective vector group. MFN2, mitofusin 2 ; si, small interfering; $\mathrm{Ri}, \mathrm{RNA}$ interference.

bladder cancer cells, the expression of MFN2 was knocked down using specific siRNA in T24 and J82 cell lines (Fig. 2A). The BrdU incorporation assay demonstrated that knockdown of MFN2 significantly increased cell proliferation in both cell lines (Fig. 2B). Furthermore, wound healing and Transwell assays revealed that MFN2 silencing significantly increased cell migration and invasion in bladder cancer cells (Fig. 2C and D).

MFN2 knockdown facilitates the tumorigenicity of bladder cancer cells in vivo. To examine the role of MFN2 in 
A

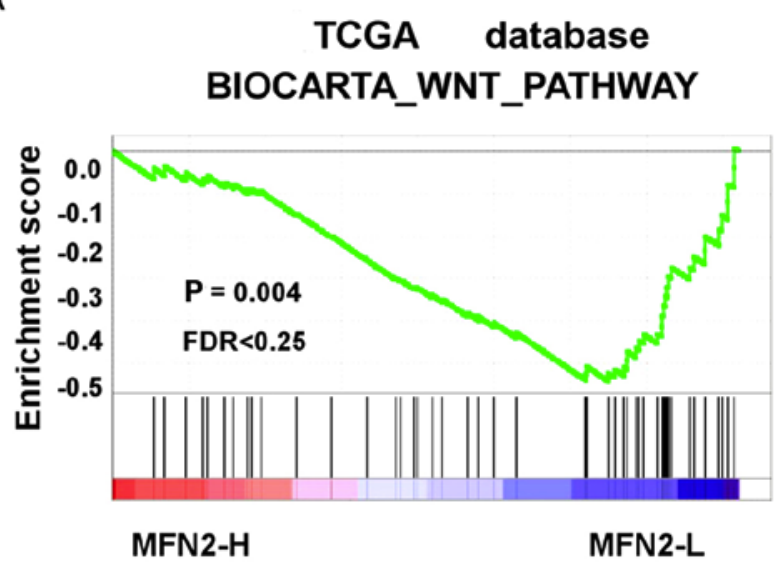

B

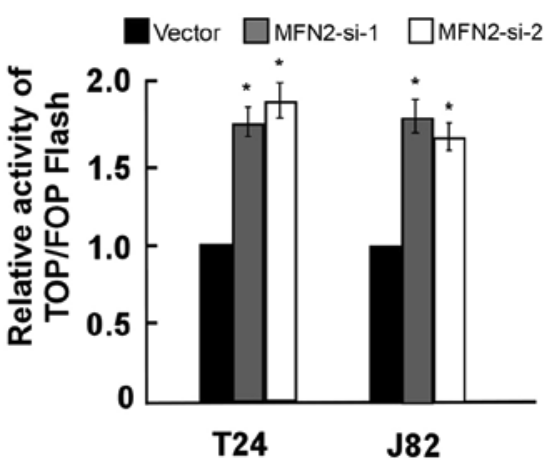

C

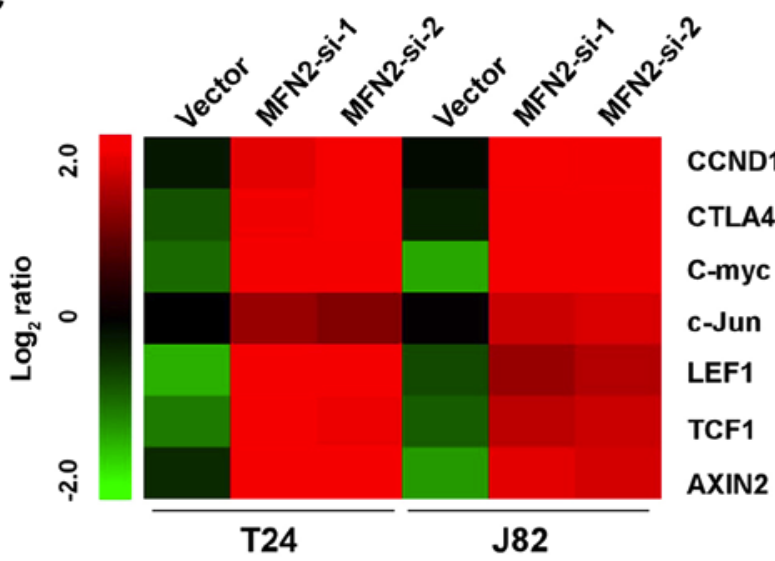

D

T24

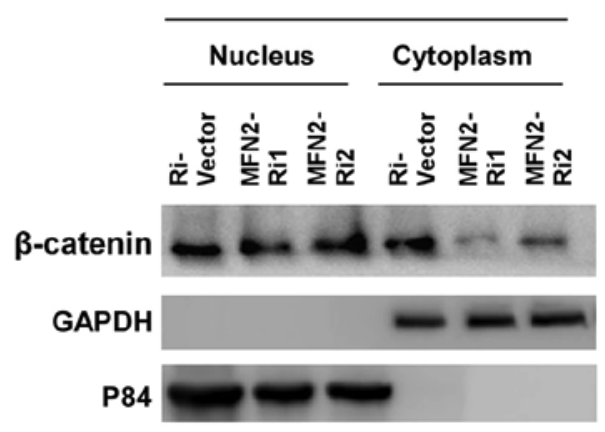

J82

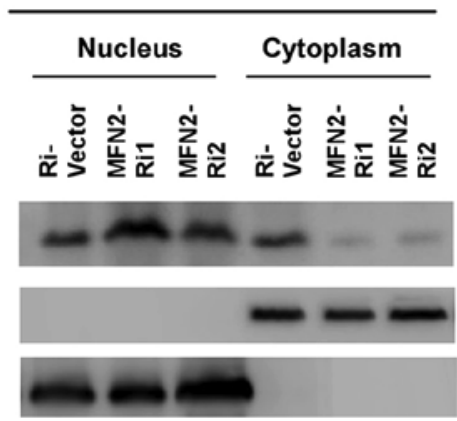

Figure 4. MFN2 knockdown activates the Wnt/ $\beta$-catenin signaling pathway. (A) A gene set enrichment analysis plot demonstrated that MFN2 expression is negatively associated with Wnt/ $\beta$-catenin-activated gene signatures in published bladder cancer gene expression profiles. (B) TOP/FOP luciferase assay of TCF/LEF transcriptional activity in the indicated cells. (C) Reverse transcription-quantitative polymerase chain reaction analysis of the expression of genes downstream of the Wnt signaling pathway, including CCND1, CTLA4, c-myc, c-Jun, LEF1, TCF1 and Axin2. (D) Western blot analysis of $\beta$-catenin expression levels in the cytoplasm and nucleus of the indicated cells. GAPDH and p84 were used as loading controls for the cytoplasmic and nuclear fractions, respectively. Data are presented as the mean \pm standard deviation of three independent experiments. " $\mathrm{P}<0.05$ vs. respective vector group. MFN2, mitofusin 2 ; TCGA, The Cancer Genome Atlas; H, high; L, low; FDR, false discovery rate; si, small interfering; Ri, RNA interference; CCND1, cyclin D1; CTLA4, cytotoxic T-lymphocyte associated protein 4; LEF1, lymphoid enhancer binding factor 1; TCF1, T-cell factor 1.

the tumorigenicity of bladder cancer cells in vivo, subcutaneous xenograft tumor models were generated using MFN2-knockdown T24 and J82 cells. The results demonstrated that tumors formed from MFN2-knockdown cells were larger and significantly heavier compared with the control (Fig. 3A-C). In addition, IHC staining revealed a significantly higher expression level of $\mathrm{Ki} 67$ and an increased proliferation index in the MFN2-knockdown groups compared with the control group (Fig. 3D and E). In summary, the in vivo studies indicated that suppressed expression of MFN2 promotes tumorigenicity.

MFN2 regulates bladder cancer cell proliferation and migration via the Wnt/ $\beta$-catenin signaling pathway. The molecular mechanisms of MFN2-inhibited bladder cancer cell proliferation and migration were also investigated. Publicly 
available gene expression array data for bladder cancer was analyzed by GSEA and it was observed that MFN2 expression was negatively associated with the activation of the Wnt/ $\beta$-catenin pathway (Fig. 4A). Furthermore, knockdown of MFN2 significantly increased the transactivation activity of $\beta$-catenin/TCF in both T24 and J82 cells, which was revealed by luciferase reporter assay (Fig. 4B). Furthermore, the expression levels of downstream genes of $\mathrm{Wnt} / \beta$-catenin, including cyclin D1, cytotoxic T-lymphocyte associated protein 4, c-myc, c-Jun, lymphoid enhancer binding factor 1, T-cell factor 1 and Axin2, were markedly higher in MFN2-knockdown bladder cancer cells compared with control cells (Fig. 4C). Notably, knockdown of MFN2 increased the translocation of $\beta$-catenin into the nucleus, as determined by immunoblotting of cytoplasmic and nuclear protein extracts (Fig. 4D).

\section{Discussion}

The present study investigated the clinical significance and biological role of MFN2 in bladder cancer pathogenesis. First, the expression level of MFN2 was revealed to be significantly lower in bladder cancer tissue compared with adjacent non-tumor tissues. Second, MFN2 expression level was identified to be associated with tumor stage, lymph node metastasis and poor prognosis in patients with bladder cancer. Third, silencing of MFN2 promoted bladder cancer proliferation and metastasis via regulation of the $\mathrm{Wnt} / \beta$-catenin signaling pathway. These results indicated that MFN2 is a candidate tumor suppressor in bladder cancer, and may be exploited as a target for potential clinical treatments for bladder cancer.

MFN2, also termed the hyperplasia suppressor gene, was originally identified in vascular smooth muscle cells from spontaneously hypertensive rats (15). Overexpression of MFN2 induces the formation of mitochondrial networks and may involve a major rearrangement of the coiled coil domains (16). It is also involved in the clearance of damaged mitochondria via selective autophagy and the regulation of the unfolded protein response upon estrogen receptor stress (17).

Notably, MFN2 functions as a mitochondrial receptor for Parkin protein (PARK2) and is required for PARK2 recruitment to dysfunctional mitochondria (18). A previous study revealed the antitumor effect of MFN2 in cancer cells (19). Further studies confirmed this anti-proliferative effect in a number of tumor cell lines, including hepatocellular carcinoma cells (20), breast cancer cells (10) and lung adenocarcinoma cells (21). However, to the best of our knowledge, the expression of MFN2 in bladder cancer tissues, and its potential clinical and biological significance, have not been described. The present study identified that MFN2 expression was significantly downregulated in bladder cancer tissues and cells compared with the control groups, which indicated that MFN2 may function as a tumor suppressor in bladder cancer.

A number of studies have demonstrated that MFN2 expression is tightly associated with the clinical prognosis of patients with cancer. Wang et al (20) reported that patients with hepatocellular carcinoma with low MFN2 expression exhibited a poorer prognosis compared with patients with high MFN2 expression. In support of these findings, Feng et al (22) demonstrated that semaphorin $3 \mathrm{C}$ and MFN2 co-expression has value for the prognosis of patients with hepatocellular carcinoma following hepatectomy. Fang et al (23) observed that patients with gastric cancer with high expression levels of MFN2 have a significantly higher overall survival rate and disease-free survival rate compared with those with low expression levels. The current study demonstrated that the MFN2 expression level was significantly associated with overall and recurrence-free survival of patients with bladder cancer. In summary, these studies suggest that MFN2 may serve as a prognostic factor for predicting the clinical outcome of patients with cancer.

In addition to a direct role in predicting clinical prognosis, studies have demonstrated that MFN2 exhibits a role in regulating tumor cell growth. Xu et al (10) demonstrated that MFN2 suppresses breast cancer cell growth and metastasis through inhibition of mTORC2/Akt signaling. By contrast, inhibition of MFN2 can effectively repress tumor growth and metastasis (24). Similarly, Ma et al (25) reported that MFN2 mediates MCF-7 cell proliferation and apoptosis via the phosphoinositide 3-kinase/Akt signaling pathway. The current results demonstrated that MFN2 can regulate bladder cancer cell proliferation, migration and invasion through the Wnt/ $\beta$-catenin signaling pathway. However, the detailed cellular signaling mechanisms between MFN2 and Wnt signaling require further investigation. In addition, it was identified that low MFN2 expression was predictive of lymph node metastasis in patients with bladder cancer. Therefore, the present results are consistent with previous findings that suggest MFN2 may serve an important role in promoting cancer migration and progression. However, further studies are required to investigate the precise role of MFN2 in the metastatic process of bladder cancer.

The present study possessed several limitations that should be acknowledged. First, it was a small retrospective review at a single center, which included only 117 patients with MIBC. Second, only the effects of MFN2 knockdown in bladder cancer cells were examined and the effects of MFN2 overexpression in other cell lines were not investigated.

In conclusion, MFN2 may act as an independent marker for the prognosis of patients with bladder cancer. Silencing of MFN2 can promote cell proliferation, migration and invasion. Therefore, MFN2 may be used as a potential therapeutic target for bladder cancer in the future.

\section{Acknowledgements}

Not applicable.

\section{Funding}

This study was supported by the Science and Technology Planning Project of Zhuhai People's Hospital (grant no. 2009402970).

\section{Availability of data and materials}

All data analyzed during this study are included in this manuscript.

\section{Authors' contributions}

GP and QX conducted the experiments and analyzed the results. GP and JY designed the study and wrote the manuscript. All 
authors reviewed the results and approved the final version 2of the manuscript.

\section{Ethics approval and consent to participate}

The present study was approved by the Institutional Review Board of Zhuhai People's Hospital (Zhuhai, China). Written informed consent was obtained from all participants. All animal care and experiments were approved by the Institutional Animal Care and Use Committee of Zhuhai People's Hospital.

\section{Patient consent for publication}

Not applicable.

\section{Competing interests}

The authors declare that they have no competing interests.

\section{References}

1. Popovic LS, Matovina-Brko G and Popovic M: Checkpoint inhibitors in the treatment of urological malignancies. ESMO open 2: e000165, 2017.

2. Siddiqui MR, Grant C, Sanford T and Agarwal PK: Current clinical trials in non-muscle invasive bladder cancer. Urol Oncol 35 516-527, 2017.

3. Zlatev DV, Altobelli E and Liao JC: Advances in imaging technologies in the evaluation of high-grade bladder cancer. Urol Clin North Am 42: 147-157, vii, 2015.

4. Bellmunt J, Orsola A, Leow JJ, Wiegel T, De Santis M and Horwich A; ESMO Guidelines Working Group: Bladder cancer: ESMO Practice Guidelines for diagnosis, treatment and follow-up. Ann Oncol 25 (Suppl 3): iii40-iii48, 2014.

5. Papanicolaou KN, Kikuchi R, Ngoh GA, Coughlan KA, Dominguez I, Stanley WC and Walsh K: Mitofusins 1 and 2 are essential for postnatal metabolic remodeling in heart. Circulation research 111: 1012-1026, 2012.

6. Chan DC: Mitochondria: Dynamic organelles in disease, aging, and development. Cell 125: 1241-1252, 2006

7. Guo X, Chen KH, Guo Y, Liao H, Tang J and Xiao RP: Mitofusin 2 triggers vascular smooth muscle cell apoptosis via mitochondrial death pathway. Circ Res 101: 1113-1122, 2007.

8. Bach D, Pich S, Soriano FX, Vega N, Baumgartner B, Oriola J, Daugaard JR, Lloberas J, Camps M, Zierath JR, et al: Mitofusin-2 determines mitochondrial network architecture and mitochondrial metabolism. A novel regulatory mechanism altered in obesity. J Biol Chem 278: 17190-17197, 2003

9. Zuchner S, Mersiyanova IV, Muglia M, Bissar-Tadmouri N, Rochelle J, Dadali EL, Zappia M, Nelis E, Patitucci A, Senderek J, et al: Mutations in the mitochondrial GTPase mitofusin 2 cause Charcot-Marie-Tooth neuropathy type 2A. Nat Genet 36: 449-451, 2004.

10. Xu K, Chen G, Li X, Wu X, Chang Z, Xu J, Zhu Y, Yin P, Liang X and Dong L: MFN2 suppresses cancer progression through inhibition of mTORC2/Akt signaling. Sci Rep 7: 41718, 2017.
11. Zhang GE, Jin HL, Lin XK, Chen C, Liu XS, Zhang Q and Yu JR: Anti-tumor effects of Mfn2 in gastric cancer. Int J Mol Sci 14: 13005-13021, 2013.

12. Wang G and McKenney JK: Urinary Bladder Pathology: World Health Organization (WHO) Classification and American Joint Committee on Cancer (AJCC) Staging Update. Arch Pathol Lab Med 143: 571-577, 2018.

13. Livak KJ and Schmittgen TD: Analysis of relative gene expression data using real-time quantitative PCR and the 2(-Delta Delta C(T)) method. Methods 25: 402-408, 2001.

14. Cui Y, Ma W, Lei F, Li Q, Su Y, Lin X, Lin C, Zhang X, Ye L, Wu S, et al: Prostate tumour overexpressed-1 promotes tumourigenicity in human breast cancer via activation of Wnt/beta-catenin signalling. J Pathol 239: 297-308, 2016.

15. Chen G, Liu N, Zhou A, Tang C, Ma D and Tang J: The role of hypertension-related gene in aortic vascular smooth muscle cells from mice and rats. Chin Med J (Engl) 114: 833-836, 2001.

16. Cao YL, Meng S, Chen Y, Feng JX, Gu DD, Yu B, Li YJ, Yang JY, Liao S, Chan DC and Gao S: MFN1 structures reveal nucleotide-triggered dimerization critical for mitochondrial fusion. Nature 542: 372-376, 2017.

17. Abdelwahid E, Li H, Wu J, Irioda AC, de Carvalho KA and Luo X: Endoplasmic reticulum (ER) stress triggers Hax1-dependent mitochondrial apoptotic events in cardiac cells. Apoptosis 21: 1227-1239, 2016.

18. Chen Y and Dorn GW II: PINK1-phosphorylated mitofusin 2 is a Parkin receptor for culling damaged mitochondria. Science 340: 471-475, 2013.

19. Jin B, Fu G, Pan H, Cheng X, Zhou L, Lv J, Chen G and Zheng S: Anti-tumour efficacy of mitofusin-2 in urinary bladder carcinoma. Med Oncol 28 (Suppl 1): S373-S380, 2011.

20. Wang W, Xie Q, Zhou X, Yao J, Zhu X, Huang P, Zhang L, Wei J, Xie H, Zhou L and Zheng S: Mitofusin-2 triggers mitochondria $\mathrm{Ca}^{2+}$ influx from the endoplasmic reticulum to induce apoptosis in hepatocellular carcinoma cells. Cancer Lett 358: 47-58, 2015.

21. Lou Y, Li R, Liu J, Zhang Y, Zhang X, Jin B, Liu Y, Wang Z, Zhong H, Wen S and Han B: Mitofusin-2 over-expresses and leads to dysregulation of cell cycle and cell invasion in lung adenocarcinoma. Med Oncol 32: 132, 2015.

22. Feng X, Zhu K, Liu J, Chen J, Tang J, Liang Y, Jin R, Liang X and Cai X: The evaluative value of Sema3C and MFN2 co-expression detected by immunohistochemistry for prognosis in hepatocellular carcinoma patients after hepatectomy. Onco Targets Ther 9: 3213-3221, 2016.

23. Fang CL, Sun DP, Chen HK, Lin CC, Hung ST, Uen YH and Lin KY: Overexpression of Mitochondrial GTPase MFN2 represents a negative prognostic marker in human gastric cancer and its inhibition exerts anti-cancer effects. J Cancer 8: 1153-1161, 2017.

24. Zhou X, Zhang L, Zheng B, Yan Y, Zhang Y, Xie H, Zhou L, Zheng S and Wang W: MicroRNA-761 is upregulated in hepatocellular carcinoma and regulates tumorigenesis by targeting Mitofusin-2. Cancer Sci 107: 424-432, 2016.

25. Ma LI, Chang Y, Yu L, He W and Liu Y: Pro-apoptotic and anti-proliferative effects of mitofusin-2 via PI3K/Akt signaling in breast cancer cells. Oncol Lett 10: 3816-3822, 2015 .

This work is licensed under a Creative Common Attribution-NonCommercial-NoDerivatives 4.0 International (CC BY-NC-ND 4.0) License. 